\title{
Teoria de Redes de Influências de Stakeholders: uma abordagem revisitada
}

\author{
FABRICIO STOCKER ${ }^{1}$ \\ Keysa Manuela Cunha Mascena ${ }^{2}$ \\ ANA CLÁUdia AZEVEdo ${ }^{1}$ \\ JoÃo MAURícIo GaMA BoAVENTURA ${ }^{1}$
}

\author{
${ }^{1}$ Universidade de São Paulo (USP) / Faculdade de EConomia, Administração e Contabilidade, SÃo PAULO - SP, BRASIL
}

2 UNIVERSIDADE DE FORTALEZA (UNIFOR), FORTALEZA - CE, BRASIL

\begin{abstract}
Resumo
As interações entre múltiplos stakeholders vêm ganhando importância nas últimas décadas, dada a velocidade com que se propagam informações e se estabelecem conexões entre indivíduos e grupos. Porém, a pesquisa sobre o tema ainda carece de estudos empíricos que analisem o fenômeno das redes de stakeholders e da sua contribuição para o avanço da teoria. Assim, este trabalho tem como objetivo investigar a evolução da abordagem de redes de stakeholders nos últimos 20 anos e fornecer um panorama da sua evolução até os dias atuais. Este trabalho fundamenta-se em dois escopos de pesquisa: Teorias de Redes - sociedade em redes e redes interoganizacionais e a Teoria de Stakeholders, integradas na composição da proposta de Rowley (1997) -; Teoria de Redes de Stakeholders. Valendo-se da análise sociométrica e da revisão sistemática de 228 artigos coletados no Web of Science entre 1997 e 2017, foi possível analisar a evolução desta abordagem. Inferimos, por esta reflexão, que o interesse das pesquisas recentes deslocou-se das relações com os Stakeholders centradas na organização para as relações em rede descentralizadas e com diversos atores. Além disso, há uma tendência de estudos das redes formadas por grupos de stakeholders e estudos das identidades sociais dos indivíduos membros desses grupos. A contribuição deste estudo consiste em fornecer um panorama da Teoria de Redes de stakeholders, analisando as redes de referências, as contribuições teóricas e empíricas, as tendências de pesquisa, além de sugerir tópicos de pesquisa para trabalhos futuros. Palavras-chave: Múltiplos stakeholders. Redes interorganizacionais. Teoria dos stakeholders. Influência das partes interessadas. Análise sociométrica.
\end{abstract}

\section{Network theory of stakeholder influences: a revisited approach}

\section{Abstract}

The interactions among multiple stakeholders have gained importance in the last decades, given the speed information spreads and connections established between individuals and groups. However, there is still a research gap, which is the lack of consolidation of the empirical studies that analyzed the phenomenon of stakeholder networks and their contribution to the advancement of the theory. This work aims to investigate the evolution of the stakeholder networks approach in the last 20 years, that is, to revisit Rowley's theory (1997) and to bring a panorama of its evolution to the present day. This essay is based on two research scopes: Network Theories- society in networks and interoganizational networks and Theory of Stakeholders, integrated into the composition of Rowley's proposal (1997)- Network Theory of Stakeholders Influences. From a sociometric analysis and systematic review of 228 articles collected from the Web of Science database between 1997 and 2017 , it was possible to analyze the evolution of this approach. The study indicates that recent research shifts the focus of the relations with the stakeholders, centered in the organization, to those of a decentralized network with several actors. In addition, there is a trend of studies of networks formed by groups of stakeholders and the social identities of individuals members of these groups. This study contributes to the field by presenting an overview of the Network Theory of Stakeholder Influences analyzing reference networks, theoretical and empirical contributions, trends and research agenda for the theme, which will help in the development of future works.

Keywords: Multiple Stakeholders. Interorganizational Networks. Stakeholder theory. Stakeholder influences. Sociometric analysis.

\section{Teoría de redes de influencia de stakeholders: un enfoque revisado}

\section{Resumen}

Las interacciones entre múltiples stakeholders han ganado importancia en las últimas décadas, dada la velocidad con la que se difunde la información y se establecen conexiones entre individuos y grupos. Sin embargo, todavía hay una brecha en la investigación, que es la falta de consolidación de estudios empíricos que analicen el fenómeno de las redes de stakeholders y su contribución al avance de la teoría. Por lo tanto, este trabajo tiene como objetivo investigar la evolución del enfoque de redes de stakeholders en los últimos 20 años, es decir, revisar la teoría de Rowley (1997) y presentar un panorama de su evolución hasta el presente. Este ensayo se basa en dos ámbitos de investigación: Teorías de redes -sociedad en redes y redes interorganizacionales y Teoría de Stakeholders (partes interesadas), integradas en la composición de la propuesta de Rowley (1997)- y, Teoría de redes de las influencias de stakeholders. A partir de un análisis sociométrico y una revisión sistemática de 228 artículos recopilados en Web of Science entre 1997 y 2017, fue posible analizar la evolución de este enfoque. Sobre la base de esta reflexión, es posible inferir que las investigaciones recientes cambian el enfoque de las relaciones con las partes interesadas, centradas en la organización, a las de una red descentralizada y con varios actores. Además, hay una tendencia de estudios de redes formadas por grupos de partes interesadas y las identidades sociales de los miembros individuales de estos grupos. La contribución de este estudio consiste en presentar un panorama de la teoría de redes de stakeholders analizando las redes de referencia, las contribuciones teóricas y empíricas, las tendencias de investigación, además de sugerir temas de investigación para trabajos futuros.

Palabras clave: Múltiples stakeholders. Redes interorganizacionales. Teoría de los stakeholders. Influencia de las partes interesadas. Análisis sociométrico. 


\section{INTRODUÇÃO}

Pesquisadores e profissionais da área de estratégia reconhecem que a cooperação é fundamental para o sucesso organizacional. As organizações podem ser consideradas como um sistema de cooperação e de esforços coordenados para suas atividades fins (BARNARD, 1938). Desde o final da década de 1930, os processos de cooperação vêm sendo pesquisados, especialmente no que concerne ao campo intra e interorganizacional (GIBBS e SINGER, 1993), às redes (POWELL, 1990), e às alianças organizacionais (CONTRACTOR e LORANGE, 1988).

Na teoria de stakeholders, a maioria das pesquisas sobre cooperação tem sido focada no que é conhecido como o campo de colaboração interorganizacional (GRAY e WOOD, 1991; HUXHAM e VANGEN, 1996; ROBERTS e BRADLEY, 1991). Ou seja, a relação com os stakeholders tem sido centrada no nível diádico, excluindo as múltiplas e complexas estruturas das empresas. Os resultados relacionais, emergentes dos laços diretos e indiretos entre a empresa e seus stakeholders, são observados apenas sob a estrutura da relação em via única entre a empresa e cada um de seus stakeholders (HEUGENS, VAN DEN BOSCH e VAN RIEL, 2002; ROWLEY, 1997).

Os resultados relacionais são importantes para se analisar o comportamento das empresas e de seus stakeholders, pois, as empresas não respondem a cada interessado de forma individual, mas, sim, em consonância com as múltiplas influências de todo um conjunto de stakeholders (NEVILLE e MENGUC, 2006). Esse fenômeno caracteriza as chamadas redes de stakeholders, e sua análise pode evidenciar como os resultados relacionais são obtidos por meio das interações entre as organizações e suas múltiplas partes interessadas.

Portanto as redes de stakeholders têm sua relevância como campo de estudos, considerando-se que a visão tradicional do relacionamento da empresa com seus stakeholders não é suficiente para explicar a complexidade desses relacionamentos. Os stakeholders relacionam-se com base em interesses compartilhados, têm capacidades coletivas de influência na estratégia da empresa (FROOMAN, 2010; SCHNEIDER e SACHS, 2017), assumem múltiplos papéis com relação a uma organização, e estão interligados em um sistema de criação de valor (HARRISON, FREEMAN e ABREU, 2015).

A abordagem teórica precursora nesse campo de estudos é a de Rowley (1997), que propõe a Network Theory of Stakeholder. Por meio da análise de rede social, Rowley (1997) avalia como se dá o relacionamento entre os múltiplos stakeholders, indo além das relações diádicas entre eles e uma organização central. Mesmo havendo importância teórica e prática para o estudo das relações entre os múltiplos stakeholders, a abordagem teórica proposta não demonstrou relativa evolução e aplicação ao longo dos últimos anos (ROWLEY, 2017).

Considerando-se a atual dinâmica de propagação de informações e de estabelecimento de conexões entre indivíduos e grupos, evidencia-se que a importância das interações entre os stakeholders também tenha crescido nas últimas décadas. Contudo faltam ainda estudos empíricos que analisem o fenômeno das redes de stakeholders e contribuam para o avanço da teoria. Correlativamente, também se fazem necessárias a atualização e a revigoração das premissas teóricas que fundamentem a pesquisa do campo.

Portanto este trabalho tem como objetivo investigar a evolução da abordagem de redes de stakeholders, por meio de uma análise sociométrica e de uma revisão sistemática das publicações sobre o tema nos últimos 20 anos, desde a sua concepção em 1997. Elaboramos um panorama da abordagem teórica de redes sociais e de stakeholders que apresenta: as redes de referências, as contribuições teóricas e empíricas e a tendência dos estudos recentes. Assim, esta pesquisa contribui para o debate atual sobre o tema, além de auxiliar em futuras pesquisas.

\section{FUNDAMENTAÇÃO TEÓRICA}

O conceito de redes origina-se de estudos da sociologia e da antropologia (JACK, 2010). A abordagem do conceito, bastante extensa, associa-se a temáticas transversais conforme os diferentes campos da literatura especializada, e gera diferentes concepções, como a de redes sociais, a de redes organizacionais, e até mesmo a de redes de stakeholders (PROVAN, FISH e SYDOW, 2007), particularmente visada neste trabalho. 


\section{A abordagem das redes sociais}

Network, segundo Scott e Davis (2007), consiste em "nós" e "laços", ou as relações entre os nós. Para os autores, os "nós" são representados em uma rede por seus atores, como pessoas, grupos de indivíduos, organizações ou entidades. Assim como a sociedade é constituída por uma grande rede de interconexões (CASTELLS, MAJER e GERHARDT, 2002), no contexto das organizações, a dinâmica das relações também se dá na forma das redes interorganizacionais.

Avanços na metodologia permitiram que a metáfora de redes pudesse se tornar um método formal de análise, no qual novos constructos e medidas foram introduzidos, sistemas mais rigorosos foram estabelecidos para testar as teorias, assim como foram elaboradas novas maneiras de considerar as organizações, suas estruturas e suas relações.

Uma das premissas da teoria de redes sociais, segundo Scott e Davis (2007), refere-se ao fato de que uma rede é um sistema de relações entre partes, e as partes são geralmente referidas como "nós", e as relações ou conexões entre estes "nós" são os "laços". Nessa perspectiva, a premissa é de que os laços influenciam mais o comportamento dos atores da rede do que os próprios atributos específicos dos nós. Além disso, o comportamento de um nó é influenciado não somente pelos laços aos quais ele está diretamente envolvido, como também pela padronização de laços na rede.

Considerando a dinâmica das redes sociais, Granovetter (1985) defende a sua proposta de embeddedness, termo traduzido como imersão, na qual a compressão sobre os comportamentos e as instituições a serem analisados deve ser ponderada pelas contínuas relações sociais em que estão imersas. Portanto, interpretá-los como elementos independentes representa um grave mal-entendido, segundo o autor.

Assim, os estudos organizacionais nos últimos anos passaram a se ocupar também dos relacionamentos entre os indivíduos, tendo em conta os modos das relações sociais, seja numa perspectiva mais ampla, seja individual (GRANOVETTER, 2017).

Nesse aspecto, é importante ressaltar que, com o advento tecnológico, as redes sociais se reconfiguraram. A estrutura sobre a qual se alicerça a sociedade em rede (CASTELLS, 1999) foi potencialmente ampliada com o avanço das tecnologias de comunicação e de informação, possibilitando novas perspectivas sobre o fenômeno, a exemplo configurando as redes como redes sociotécnicas.

Esses "novos arranjos" são descritos como redes em que a tecnologia oferece a estrutura de sustentação das relações sociais que se estabelecem ou se estabelecerão (LATOUR, 1994). As redes sociotécnicas são como um novo canal, mais ágil e complexo, o qual, guardadas as suas peculiaridades, configura-se em um espaço fértil para viabilizar a produção e a circulação de conhecimento, e a emergência de novas formas sociais (CALLON, 2004).

\section{A abordagem das redes organizacionais}

Na medida em que o ambiente econômico se tornou global, tecnologicamente conectado e fortemente mais competitivo, novas configurações organizacionais emergiram, e as redes assumiram uma crescente importância estratégica (GULATI, NOHRIA e ZAHEER, 2000). A evolução e o contínuo crescimento desses arranjos originaram um grande campo de pesquisa, voltado para a análise das relações dentro e entre as organizações (AHUJA, SODA e ZAHEER, 2012), pauta claramente em voga na literatura (BERGENHOLTZ e WALDSTR $\varnothing M$, 2011).

No âmbito geral, as redes organizacionais referem-se às interações entre um conjunto de organizações que ultrapassam suas fronteiras organizacionais. Nesse sentido, Gulati, Nohria e Zaheer (2000) argumentam que as redes englobam um conjunto de relações horizontais e verticais de uma empresa com outras organizações, sejam elas de fornecedores, de clientes, de concorrentes ou de outras entidades, e são compostas de laços interorganizacionais duradouros, com um significado estratégico para as empresas.

Pesquisas sobre o tema de redes organizacionais têm aumentado em diversas áreas do conhecimento, como a Sociologia, a Economia e a Administração (PROVAN, FISH e SYDOW, 2007). As redes organizacionais promovem o compartilhamento de conhecimentos, competências e informações, recursos primordiais para a competitividade e para a inovação (BALESTRIN e VERSCHOORE, 2016). Assim, ao trabalhar em rede, as organizações podem alcançar níveis de desempenho os quais elas dificilmente alcançariam sozinhas (ZACCARELLI, TELLES, SIQUEIRA et al., 2008).

Com o fenômeno da globalização, novas estruturas organizacionais foram formadas e a forma de organização em redes ganhou ainda mais importância. Atuando como intermediárias organizacionais, as redes têm como finalidade formar ambientes para que certas organizações consigam superar a complexidade estratégica de outras (CASAROTTO FILHO e PIRES, 1998). 
Apesar das diversas tipologias das redes organizacionais, um denominador comum entre todas é que elas constituem-se por meio de processos relacionais complexos e dinâmicos, regidos por fatores estratégicos de competição e de cooperação, que caracterizam uma interdependência relacional entre as organizações (ROTH, WEGNER, ANTUNES JÚNIOR et al., 2012). Algumas redes podem ser descentralizadas ou não ter uma estrutura organizacional bem definida, e outras podem ser rigidamente organizadas com altos níveis de centralização e controle. Mas, em ambos os cenários, estes arranjos são relacionados à flexibilidade da forma organizacional, tendo em vista que os objetivos sejam alcançados, sem que seja necessária a uniformização entre os grupos e as organizações integrantes à rede (CASTELLS, 1999).

Dessa maneira, pode-se afirmar que as organizações estão envolvidas em redes organizacionais, com diferentes graus de centralização e de formalidade, e que a forma organizacional baseada em relações em rede possibilita uma melhor orientação e desenvolvimento de estratégias corporativas (POWELL, 1990).

Vale mencionar que a complexidade do ambiente organizacional pode eventualmente tornar obsoleta a abordagem diádica do estudo das relações entre atores da rede (YANG e BENTLEY, 2017). Na perspectiva dos stakeholders, a abordagem diádica pode não acompanhar as relações dinâmicas que constituem a sociedade (CASTELLS, 2007). Assim, para se entender a influência dos stakeholders, devem-se incorporar os múltiplos relacionamentos e descrever mais precisamente as estruturas necessárias para promover o gerenciamento dos stakeholders (YANG e BENTLEY, 2017).

\section{A abordagem de redes de stakeholders}

Tanto na teoria clássica sobre stakeholders, como nos modelos gerenciais atuais, a gestão estratégica é sempre apresentada na perspectiva de uma organização focal como centro de uma rede de stakeholders (ROWLEY, 1997). Porém, como as empresas competem em mercados complexos e interconectados com os mesmos stakeholders, uma rede maior com vários pontos focais pode ser construída (ROLOFF, 2008).

Essa abordagem originou-se em virtude da interação não apenas entre os diferentes stakeholders com uma empresa focal. Ou seja, além da relação da empresa com os stakeholders (FREEMAN e EVAN, 1990), há também a relação entre os stakeholders, que leva as empresas a não responderem individualmente a cada um deles, mas sim à influência do conjunto desses relacionamentos (ROWLEY, 1997).

Seguindo a mesma linha de raciocínio que Rowley, Yang e Bentley (2017) afirmam que as corporações nem sempre são o nexo de interações, e em vez disso, as redes sociais internas e externas entre os stakeholders podem afetar os comportamentos das organizações. Nesse sentido, Rowley (1997, p. 892), em seu trabalho inicial, já argumentava que "[...] a organização focal é mais do que simplesmente o ponto central de seus próprios stakeholders: é também um stakeholder de muitos outros pontos focais em seu sistema de interação social".

A importância da análise social da integração em rede de stakeholders vem responder a uma limitação da teoria de stakeholders, a qual se concentra mais nas relações entre a organização central e seus stakeholders, e menos na relação entre os próprios stakeholders e no grau de influência mútua nas decisões uns dos outros, o que consequentemente pode influenciar nos resultados da organização. Por essa razão, Rowley (1997) argumenta que, em muitos casos, os principais influenciadores nem sempre estão diretamente vinculados a uma organização, e que alguns stakeholders podem estar ligados à organização por intermédio de outras partes, como por meio de uma ponte ou uma interligação.

Diante desse quadro, vários pesquisadores reconhecem a importância de uma análise aprofundada das relações entre os stakeholders (ROWLEY e MOLDOVEANU, 2003; ROLOFF, 2008; GARRIGA, 2009; ZENG, CHEN, DONG et al., 2015; CABRAL, FERNANDES e RIBEIRO, 2016; ZEDAN e MILLER, 2017; YANG e BENTLEY, 2017). Eles sugerem a análise de rede social para avaliar como a estrutura da rede de stakeholders e a posição de cada stakeholder dentro dessa estrutura podem influenciar a organização.

Conforme Rowley (1997), o vínculo entre os envolvidos é evidente, uma vez que o comportamento de uma organização e a resistência às demandas de seus stakeholders são influenciados pela densidade da rede de seus stakeholders e pela sua localização dentro dessa rede. Isso se deve em parte (i) à maior facilidade e intensidade de troca de informações à medida que a rede se torna mais densa, e também (ii) à disseminação de normas, comportamentos e expectativas comuns em toda a rede, o que torna mais difícil para as organizações isolar os grupos, reter informação ou formular estratégias e alianças com objetivos contrários a outros.

Segundo Miles (2017), ao explorar como os fatores estruturais restringem e moldam o comportamento dos atores de uma determinada rede de stakeholders, Rowley (1997) justapôs os conceitos de centralidade e de densidade de redes. 
Para o autor, a existência de laços densos dentro e entre os atores em uma rede de organizações facilita a transferência de conhecimentos, comportamentos e expectativas na rede. Os altos níveis de densidade, considerados de interconectividade entre os stakeholders em uma rede, estão associados aos altos níveis de coalizões e, portanto, ao alto nível de poder desses atores. Assim, a tomada de decisão e a gestão para toda a rede são envoltas por mais riscos e derivadas de ações coletivas, e não mais de um decisor centralizador.

Revisitando a própria teoria, Rowley (2017) afirma que a integração da análise de redes sociais com a pesquisa sobre stakeholders permanece limitada até o momento.

\section{PROCEDIMENTOS METODOLÓGICOS}

Este trabalho investiga a evolução da abordagem de redes de stakeholders realizando uma análise sociométrica e uma revisão sistemática das publicações sobre o tema nos últimos 20 anos. O recorte temporal foi definido considerando-se como marco inicial dessa abordagem a publicação do artigo de Timothy J. Rowley, no Academy of Management Review, intitulado "Moving beyond dyadic ties: A network theory of stakeholder influences", no ano de 1997. O recorte temporal compreende um período de 20 anos, desde a publicação do referido artigo até o ano de 2017, data em que o autor publicou uma releitura da sua teoria intitulada The Power of and in Stakeholder Networks.

A Figura 1 mostra os procedimentos metodológicos para a coleta de dados, realizada por meio de um mapeamento da literatura internacional na base de dados Web of Science (ISI), e tendo como o filtro os journals pertencentes à categoria de Business \& Management. Esta base foi escolhida por ser "[...] um dos bancos de dados mais abrangentes de periódicos revisados por pares em Ciências Sociais" (CROSSAN e APAYDIN, 2010, p. 1157), e possuir apenas conteúdo bem reconhecido e considerado relevante pelos pares (SCARINGELLA e RADZIWON, 2017).

\section{Figura 1}

\section{Matriz das etapas e procedimentos metodológicos}

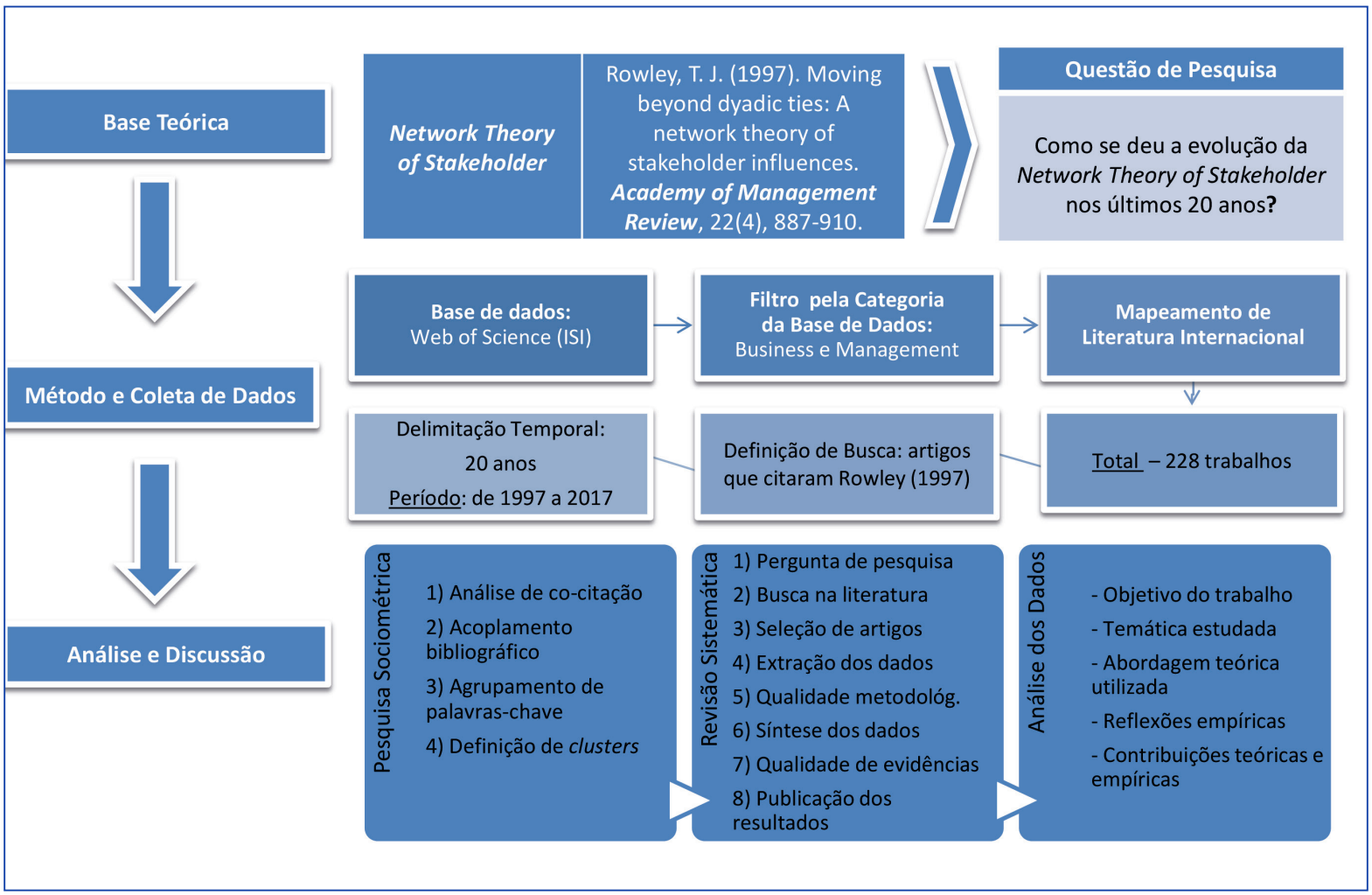

Fonte: Elaborado pelos autores. 
Foram angariados todos os artigos da referida base que citam o artigo "Moving beyond dyadicties: A network theoryof stakeholder influences", de Rowley (1997), resultando um total de 228 trabalhos. Ressalta-se que a citação em si pode representar concordância, discordância e/ou acréscimos feitos ao trabalho inicial. Portanto elas representam o interesse da revisão, efetuada neste trabalho, em mapear a evolução da teoria de Rowley.

\section{Análise sociométrica dos dados}

Inicialmente, os dados foram submetidos à análise sociométrica tendo em vista classificar os artigos de forma estrutural e organizada, e encontrar padrões e ligações entre eles. Em seguida, foram analisados os artigos mais citados ou com maior aderência ao foco do estudo, definido como a análise de redes sociais para a abordagem de stakeholders.

A análise sociométrica possibilita que se detectem as redes relacionais convertendo o qualitativo em quantitativo, e permitindo que os dados sejam tabulados e transformados em gráficos que permitem visualizar a situação individual de cada elemento no grupo (RIBEIRO, ANTONIALLI e ZAMBALDE, 2015).

A sociometria também confirma a existência de padrões característicos da organização dos grupos, suas expressões e configurações próprias (VAN ECK e WALTMAN, 2014). Os resultados das análises podem ser examinados em três níveis: no nível individual; no das relações interpessoais; e no das estruturas dos grupos. Nesta pesquisa, a análise sociométrica é empregada com o objetivo de analisar o mapa de cocitações de todos os autores resultantes da coleta de dados, e também para analisar a evolução da produção bibliográfica sobre a temática estudada, analisando as obras mais citadas e as palavras-chave mais utilizadas. O software utilizado para análise dos dados sociométricos foi o VOSviewer ${ }^{\circledR}$ versão 1.6.6.

\section{REVISÃO SISTEMÁTICA DA LITERATURA}

A revisão sistemática da literatura baseou-se em critérios pré-determinados e evidências científicas consistentes, tendo por finalidade colaborar com a escolha de estudos e/ou possibilidades analíticas e metodológicas para o desenvolvimento da questão de pesquisa levantada (SAUR-AMARAL, 2010).

O método de revisão sistemática, de acordo com Hackett e Dilts (2004), é uma metodologia rigorosa proposta para: identificar os estudos sobre um tema em questão, aplicando métodos explícitos e sistematizados de busca; avaliar a qualidade e validade desses estudos, assim como sua aplicabilidade. Ou seja, trata-se de uma investigação fundamentada em uma questão específica, em que se busca identificar, analisar e sintetizar importantes evidências disponíveis no campo. As etapas da revisão sistemática foram empreendidas nesta pesquisa conforme descrito na Figura 1.

Com relação à apresentação dos resultados dos dados analisados, o artigo está estruturado da seguinte forma: 1) descrição e análise sociométrica dos dados, com apresentação da análise de cocitação, incluindo co-citation cluster network, bibliographic coupling cluster network e ilustração do mapeamento dos clusters teóricos encontrados; 2) aplicações empíricas da Teoria da Rede de Stakeholders, com análise dos estudos empíricos que utilizam o modelo de Rowley para desenvolvimento da pesquisa, incluindo os principais resultados e pressupostos de cada estudo; 3 ) evolução teórica da abordagem de redes de stakeholders, abordando os trabalhos que citaram, discutiram e complementaram o modelo de Rowley; 4) síntese da evolução da abordagem de redes de stakeholders; 5) discussão de tendências e proposta de agenda de pesquisa.

\section{RESULTADOS E ANÁLISE DOS DADOS}

Com os procedimentos para coleta de dados, obtiveram-se 228 artigos que citam a Network Theory of Stakeholder no período de 1997 a 2017. Do total de artigos encontrados, 64 foram classificados como artigos empíricos, correspondendo a $28 \%$ da amostra, o que evidencia a ênfase desse campo de estudos no desenvolvimento teórico da abordagem de redes e stakeholders. 


\section{Descrição e análise sociométrica dos dados}

Empreendeu-se a análise sociométrica do total de 228 artigos da base de dados coletada. Por meio da sociometria, identificam-se nos artigos quais os autores mais citados em conjunto - cocitação -, o que auxilia na análise de um grande número de publicações em virtude das perspectivas teóricas adotadas. A Figura 2 apresenta o primeiro resultado da análise sociométrica, a co-citation cluster network.

Conforme observado na Figura 2, a amostra está segmentada em três agrupamentos, obtidos com o auxílio do software VOSviewer. No primeiro agrupamento (na cor vermelha), destacam-se os autores Rowley, Gulati, Powell, entre outros. No segundo agrupamento (na cor verde), destacam-se os autores Freeman, Donaldson e Phillips. No terceiro agrupamento (na cor azul), destacam-se Porter, Wood, Waddock, entre outros. Esses autores são os mais citados nas redes de co-citação, e podem ter mais de uma obra publicada no tema.

\section{Figura 2}

\section{Co-citation cluster network}

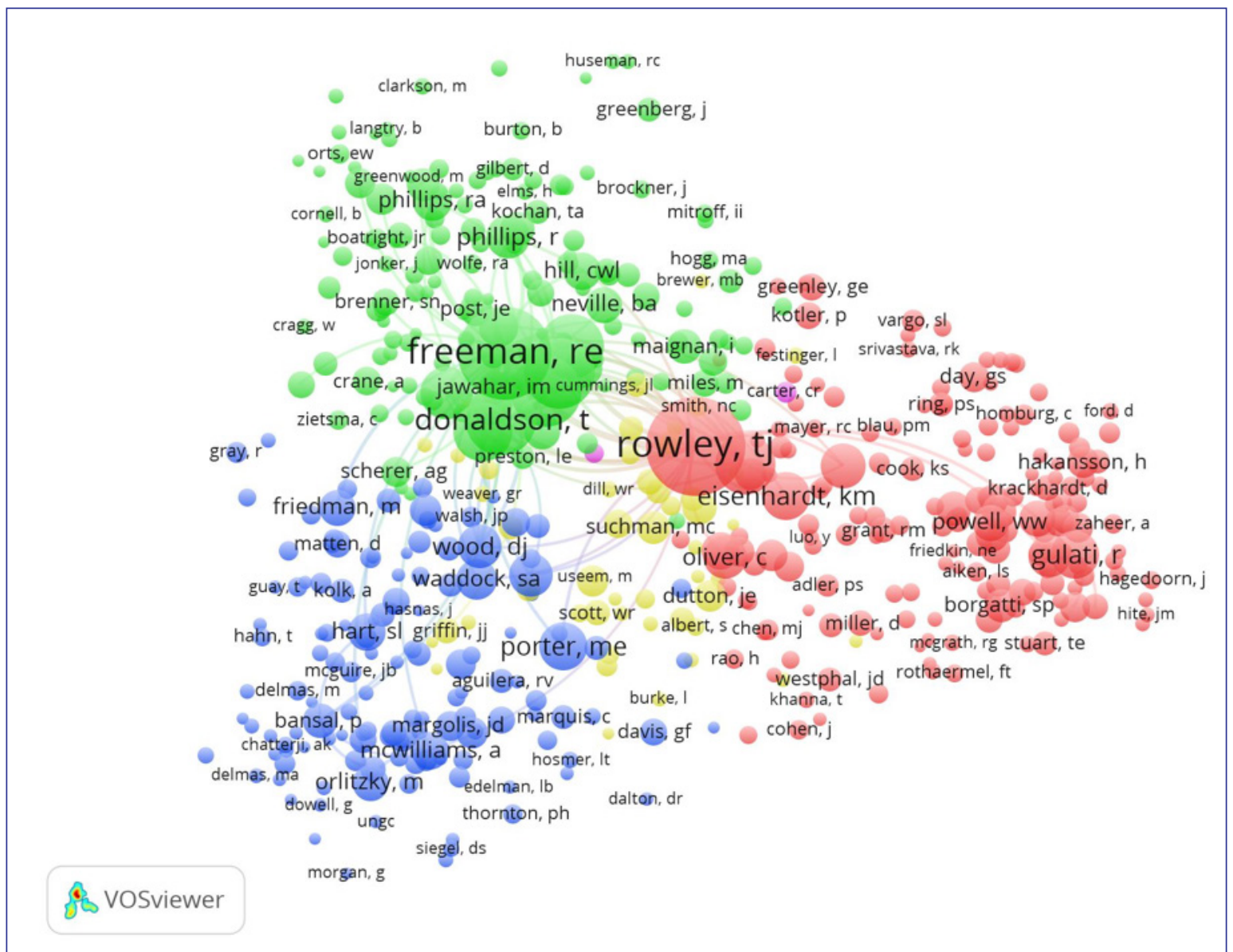

Fonte: Elaborado pelos autores.

Como complemento à análise e à identificação dos padrões de citações dos artigos analisados, elaborou-se a bibliographic coupling cluster network, que visa ao agrupamento das referências com base na obra citada. A Figura 3 apresenta o resultado da bibliographic coupling cluster network.

O acoplamento bibliográfico apresentado na Figura 3 reforça o agrupamento da amostra em três clusters. Nos dois primeiros clusters concentram-se a maior parte das citações. No primeiro agrupamento (na cor vermelha), o artigo mais citado é o de Rowley (1997); no segundo agrupamento (na cor verde), a obra mais citada é a de Freeman (1984); no terceiro agrupamento, há uma distribuição mais homogênea das citações, observando-se as obras de Barney (1991), Agle, Mitchell e Sonnenfeld (1999) e Berman, Wicks, Kotha et al. (1999), entre outros. 
Figura 3

\section{Bibliographic coupling cluster network}

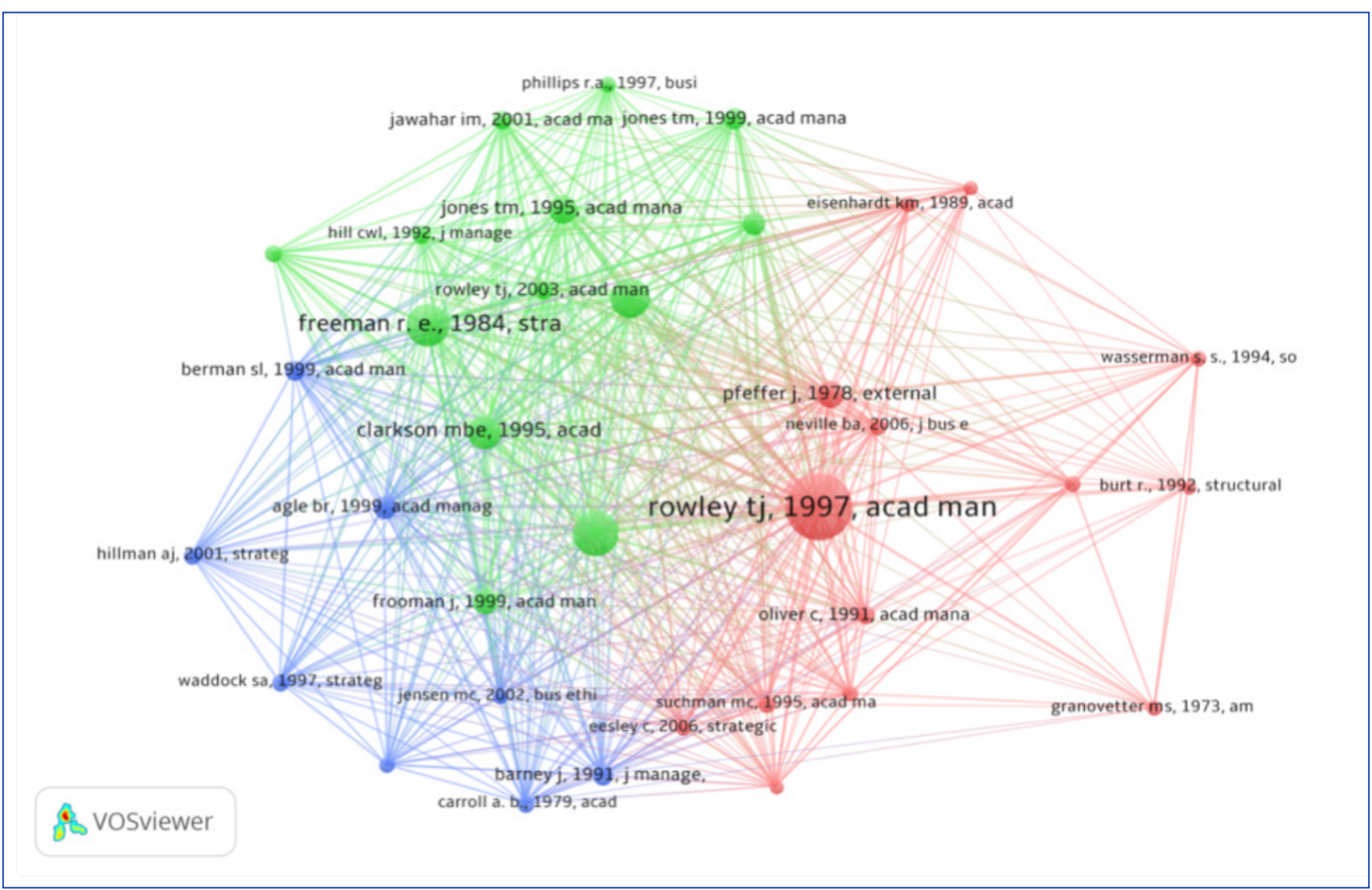

Fonte: Elaborado pelos autores.

A análise aprofundada das cocitações obtidas por meio da sociometria identificou três agrupamentos nomeados como: Cluster 1 - Social Network Theory; Cluster 2 -Stakeholder Theory; e Cluster 3 -Organizational Theory. A Figura 4 apresenta as principais citações em cada cluster, observando-se tanto a análise dos autores como a análise das obras.

Figura 4

\section{Categorização do mapeamento dos clusters teóricos}

\begin{tabular}{|c|c|c|}
\hline $\begin{array}{c}\text { Cluster 1: } \\
\text { Social Network Theory }\end{array}$ & $\begin{array}{c}\text { Cluster 2: } \\
\text { Stakeholder Theory }\end{array}$ & $\begin{array}{c}\text { Cluster 3: } \\
\text { Organizational Theory }\end{array}$ \\
\hline $\begin{array}{l}\text { - Granovetter (1985) } \\
\text {-Pfeffer e Salancik (1978) } \\
\text {-Powell (1990) } \\
\text {-Burt (1997) } \\
\text {-Rowley (1997) }\end{array}$ & $\begin{array}{l}\text { - Freeman (1984) } \\
\text {-Donaldson e Preston (1995) } \\
\text { - Clarkson (1995) } \\
\text { - Jones e Wicks (1999) } \\
\text {-Phillips (2003) } \\
\text { - Rowley e Moldoveanu (2003) }\end{array}$ & $\begin{array}{l}\text { - Porter (1980) } \\
\text { - Barney (1991) } \\
\text { - Carroll (1979) } \\
\text {-Waddock e Graves (1997) } \\
\text { - Jensen (2002) }\end{array}$ \\
\hline
\end{tabular}

Fonte: Elaborado pelos autores. 
O Cluster 1, denominado Social Network Theory, concentra as publicações fundamentadas na teoria de redes. Este agrupamento têm como temática principal de estudo as redes sociais e organizacionais, buscando compreender também a influência dos stakeholders nas organizações. O Cluster 2, denominado Stakeholder Theory, agrupa estudos que têm o framework apoiado na teoria dos stakeholders. Os artigos nesse agrupamento enfocam sua contribuição na abordagem de stakeholders e em seus diferentes meios de influenciar as organizações. O Cluster 3, denominado Organizational Theory, busca contribuir para a compreensão das vantagens competitivas das organizações, fundamentando-se também em estudos que investigam o desempenho social para os stakeholders.

Por fim, com a análise sociométrica dos agrupamentos de estudos, também é possível visualizar as principais palavraschave citadas em cada cluster, conforme descrito na Figura 4. A análise das palavras-chave reforça as características dos agrupamentos de estudo. No primeiro cluster, há uma ênfase nos estudos sobre redes de stakeholders, palavras-chave como: efeito, impacto, importância e centralidade são recorrentes. No segundo cluster, as palavras-chave remetem à teoria dos stakeholders, relacionamento com stakeholders, interesses, legitimidade e outros termos ligados à evolução dos estudos da teoria dos stakeholders. No terceiro grupo, há uma ênfase em aspectos conceituais e metodológicos, com termos como: desenho metodológico, conceitos, implicações práticas, sociedade. Formou-se também um último agrupamento com os termos desempenho, recursos, entre outros. Portanto os estudos que compõem a amostra contribuem tanto para as literaturas de redes de organizações quanto para as literaturas de stakeholders e estratégia empresarial.

Figura 5

\section{Agrupamento de palavras-chave}

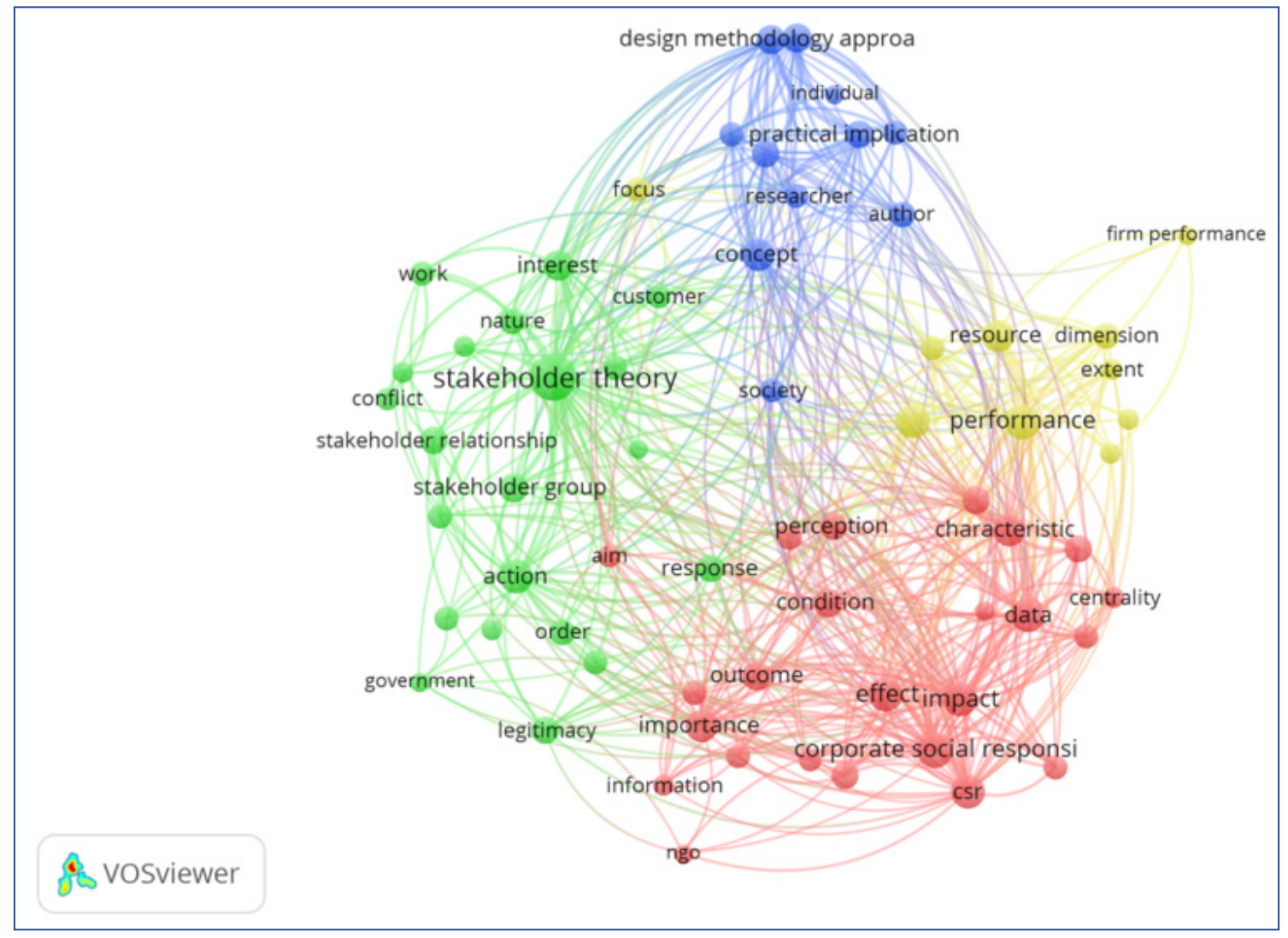

Fonte: Elaborado pelos autores.

Após a análise sociométrica dos dados, realizou-se a revisão sistemática do conjunto de publicações, observando-se: objetivos dos trabalhos, temática estudada, abordagem teórica utilizada, reflexões empíricas e contribuições resultantes. Dos 228 artigos analisados, 64 são teórico-empíricos (28\%), e, destes, 39 são estudos de casos (17\%). As contribuições dos artigos empíricos foram analisadas e observou-se que três desses estudos buscam aplicar e ampliar o modelo de Rowley (1997). Os estudos empíricos são o de Garriga (2009), o de Fassin, Colle e Freeman (2017) e o de Zedan e Miller (2017). 
Justifica-se aqui a opção pelos artigos empíricos devido ao objetivo deste trabalho de desvelar a abordagem da rede de stakeholders como prática, buscando uma compreensão de como ela se desenvolveu empiricamente nos últimos 20 anos, ou seja, desde que foi proposta.

\section{Aplicações empíricas da Network Theory of Stakeholders}

Fassin, Collee e Freeman (2017) analisam diferentes decisões de encerramento de plantas industriais em empresas multinacionais, aplicando a abordagem da teoria de stakeholders. O argumento principal do artigo baseia-se no surgimento das alianças entre stakeholders interessados em uma organização específica. A pesquisa de Fassin, Collee e Freeman (2017) foi realizada no ambiente de decisões estratégicas do setor de cervejarias multinacionais, localizadas em pequenas cidades da Itália e da Bélgica, em que houve o interesse, por parte da empresa, de encerrar as atividades empresariais. Em ambos os casos, a decisão inicial de encerramento das atividades foi revertida, graças às ações e reivindicações das alianças entre os stakeholders. Ao final, dentre outras proposições, os autores argumentam que as alianças intra-stakeholders podem ter uma influência esmagadora na estratégia corporativa, relação hipotetizada por Stocker e Mascena (2019).

Garriga (2009), partindo do gap de pesquisas empíricas que explicam o processo de cooperação utilizando a abordagem da rede de stakeholders, realizou um estudo de caso indutivo em profundidade, combinando dados sociométricos quantitativos e dados qualitativos de teoria fundamentada e observação etnográfica. Para o estudo de caso indutivo, a pesquisadora adotou o Programa de Gasoduto Natural desenvolvido em Buenos Aires, Argentina. Analisando as diversas interações entre os múltiplos stakeholders e uma determinada organização focal, Garriga (2009) conclui que há sobreposição dos interesses dos stakeholders, formados por pluralidades de indivíduos que ocupam diferentes papéis sociais e pertencem a diferentes grupos de stakeholders. Somente por meio da abordagem de redes sociais seria possível fazer essas interlocuções e entender a influência desses múltiplos atores nas decisões e estratégias das empresas, o que se alinha aos argumentos de Rowley (1997) e Rowley e Moldoveanu (2003).

Outra pesquisa baseada nos conceitos e na abordagem da Teoria das Redes de Stakeholders é a de Zedan e Miller (2017), a qual busca explorar o impacto da influência dos stakeholders sobre as decisões estratégicas no setor de engenharia e eficiência energética. $\mathrm{O}$ artigo concluiu que a rede de stakeholders tem mais conectividade com as decisões estratégicas do que o esperado pelas organizações focais, e que a transparência e o compartilhamento das informações com os stakeholders sobre as decisões das empresas têm um impacto positivo na resposta que o grupo de stakeholders dá às empresas.

Os autores apresentam o mapeamento das relações sociais entre os diferentes stakeholders, e na medida em que as métricas de densidade e centralidade são aplicadas, as relações se mostram mais interconectadas, e mais influências são reveladas entre as organizações focais e seus diversos stakeholders. A análise de rede social é uma estratégia para investigar o grau de influência de cada ator, pois, utilizando-se as métricas de centralidade, observa-se como eles podem impactar uns aos outros e o nível de conexão e coesão dentro da rede. Assim, utilizando a metodologia das redes sociais para a análise de stakeholders, pode-se dizer que os stakeholders com alta centralidade são mais propensos a influenciar os outros e têm um maior poder dentro da rede (ZEDAN e MILLER, 2017).

\section{Evolução teórica da abordagem de redes de stakeholders}

A abordagem baseada em redes de stakeholders, também definida como um conjunto de atores interdependentes afetados por uma organização (SCHNEIDER e SACHS, 2017), é uma fonte relevante de recursos para criação de valor organizacional (RÜHLI, SACHS, SCHMITT et al., 2017; BRIDOUX e STOELHORST, 2016; SCHNEIDER e SACHS, 2017).

Diferentemente de outras teorias de gestão estratégica, os pesquisadores consideram que a teoria de stakeholders incorpora a criação de valor como uma das contribuições relacionais entre uma organização e seus stakeholders (FREEMAN, 1984; DONALDSON e PRESTON, 1995; HARRISON e WICKS, 2013; TANTALO e PRIEM, 2016; BRANDÃO, DIÓGENES e ABREU, 2017). Em relação à questão da criação de valor, Rowley (1997) considera que a organização, como um ator focal de uma rede de relacionamentos com múltiplos stakeholders, é capaz de acessar os recursos e capacidades de cada stakeholder, e de se envolver em um processo de criação de valor (SCIARELLI e TANI, 2013).

Neste conjunto de pesquisas existem poucas análises dos processos subjacentes de criação de valor na abordagem de redes de stakeholders (SCHNEIDER e SACHS, 2017). Especificamente, há uma necessidade de investigar as condições prévias que 
levam à cooperação e ao comportamento equitativo de indivíduos em ambientes de múltiplos stakeholders (HARRISON e WICKS, 2013).

O estudo recente apresentado por Ali (2017) também se baseia na abordagem da rede de stakeholders. 0 autor argumenta que o papel dos gestores na rede é um pouco diluído, e reconhece a agência dos stakeholders. Nessa visão, as empresas são vistas como parte de uma rede cujas densidade e centralidade decidem o quanto elas podem ser influenciadas por certos stakeholders, corroborando as afirmações de Rowley (1997). Há de se considerar também que a sobrevivência e a prosperidade organizacional dependem das expectativas dos diversos grupos de stakeholders, e não somente dos interesses da própria organização e dos gestores que dela fazem parte (GRIFFIN, 2017).

\section{Síntese da evolução da abordagem de redes de stakeholders}

Para sintetizar a evolução da abordagem de redes de stakeholders, são apresentados no Quadro 1: a) o pressuposto principal do modelo do Rowley; b) as proposições originais apresentadas no artigo seminal do autor; c) as reflexões empíricas dos trabalhos desenvolvidos utilizando o modelo; d) as reflexões teóricas sobre o modelo e possíveis complementações sobre a teoria; e, por fim, e) os highlitgths - destaques da teoria da rede de stakeholders.

\section{Quadro 1}

\section{Framework sintético da teoria de rede de stakeholders}

\begin{tabular}{|c|c|c|c|}
\hline $\begin{array}{l}\text { Pressuposto } \\
\text { Principal da Teoria } \\
\text { de Redes de }\end{array}$ & \multicolumn{3}{|c|}{$\begin{array}{l}\text { O modelo de construção de redes sociais (densidade e centralidade) propõe ir além da análise tradicional de } \\
\text { laços diádicos, e considera as influências e o impacto dos diferentes stakeholders que não têm relações diretas } \\
\text { com a empresa focal, mas que afetam o comportamento da empresa e de toda a rede (ROWLEY, 1997, p. 907). }\end{array}$} \\
\hline $\begin{array}{c}\text { Proposições de } \\
\text { Rowley (1997) }\end{array}$ & $\begin{array}{l}\text { Proposição 1: À medida que a densi- } \\
\text { dade da rede aumenta, a capacidade } \\
\text { dos stakeholders de uma organiza- } \\
\text { ção focal, de restringir as ações da } \\
\text { organização aumenta. }\end{array}$ & $\begin{array}{l}\text { Proposição 2: À medida que a centra- } \\
\text { lidade da organização focal aumenta, } \\
\text { a sua capacidade de resistir às pres- } \\
\text { sões dos stakeholders aumenta. }\end{array}$ & $\begin{array}{l}\text { Proposição 3: Em diferentes con- } \\
\text { dições de densidade e de centrali- } \\
\text { dade, a organização focal adotará } \\
\text { um papel mais ativo ou subordinado } \\
\text { quanto aos interesses e expectati- } \\
\text { vas de toda a rede de stakeholders. }\end{array}$ \\
\hline $\begin{array}{l}\text { Reflexões empíricas } \\
\text { sobre a teoria } \\
\text { de Rowley }\end{array}$ & $\begin{array}{l}\text { Somente por meio da abordagem } \\
\text { de redes sociais é possível fazer as } \\
\text { interlocuções dos papéis sociais para } \\
\text { entender a influência dos múltiplos } \\
\text { atores nas decisões e estratégias das } \\
\text { empresas (GARRIGA, 2009). }\end{array}$ & $\begin{array}{l}\text { A rede de stakeholders tem mais } \\
\text { conectividade com as decisões estra- } \\
\text { tégicas do que o esperado pelas orga- } \\
\text { nizações focais (ZEDAN e MILLER, } \\
\text { 2017). }\end{array}$ & $\begin{array}{l}\text { As alianças intra-stakeholders podem } \\
\text { ter uma influência esmagadora na } \\
\text { estratégia corporativa (FASSIN, COLLE } \\
\text { e FREEMAN, 2017). }\end{array}$ \\
\hline $\begin{array}{l}\text { Reflexões teóricas } \\
\text { sobre o modelo } \\
\text { de Rowley }\end{array}$ & $\begin{array}{l}\text { A análise social das redes de stakehol- } \\
\text { ders é uma fonte relevante de recur- } \\
\text { sos para criação de valor organiza- } \\
\text { cional (SCHNEIDER e SACHS, 2017). }\end{array}$ & $\begin{array}{l}\text { Por meio do modelo, é possível iden- } \\
\text { tificar os recursos e capacidades de } \\
\text { cada stakeholder, para então, se } \\
\text { envolver em um processo de criação } \\
\text { de valor (SCIARELLI e TANI, 2013). }\end{array}$ & $\begin{array}{l}\text { As empresas são vistas como parte } \\
\text { de uma rede cujas densidades e } \\
\text { centralidade determinam o quanto } \\
\text { elas podem ser influenciada por seus } \\
\text { stakeholders (ALI, 2017). }\end{array}$ \\
\hline Highlights da teoria & $\begin{array}{l}\text { O modelo proposto auxilia na defi- } \\
\text { nição dos limites (boundaries) da } \\
\text { rede a ser estudada. }\end{array}$ & $\begin{array}{l}\text { Os laços relacionais entre os atores } \\
\text { são canais para transferência de } \\
\text { "fluxo" de recursos e criação de valor. }\end{array}$ & $\begin{array}{l}\text { Considera-se, principalmente, como } \\
\text { o padrão das relações entre os múl- } \\
\text { tiplos atores em conjunto afeta os } \\
\text { membros da rede. }\end{array}$ \\
\hline
\end{tabular}

Fonte: Elaborado pelos autores.

Diante dos argumentos sintetizados neste framework, é necessário ponderar que, em seu trabalho seminal, Rowley (1997) considerou em suas proposições apenas as variáveis de densidade e de centralidade como influentes na interação entre a organização e seus stakeholders. Contudo, dado o advento da internet e das tecnologias de comunicação e informação de modo geral, tornou-se inviável o estudo das evoluções sociais sem considerar o impacto destes avanços tecnológicos na dinâmica interacional dos indivíduos e grupos que as produzem e as utilizam. 
Embora este aspecto não tenha emergido nos estudos revisados, é preciso considerar que elementos característicos das redes sociotécnicas (LATOUR, 1994), dada a sua capacidade de modificar os canais relacionais, reconfigurando links de conexão e fluxos de conteúdo, devem ser integrados à análise. Nesse aspecto, ressalta-se a necessidade de incluir elementos como a conectividade, o alcance e o engajamento dos atores relacionais ao se analisar a interação entre a organização e seus stakeholders. Pois, assim como a formação de redes sociais e/ou organizacionais, a formação de comunidades virtuais (redes sociotécnicas) também se baseia na interconexão e envolve compatibilidade de interesses e um processo amplo de troca e de cooperação.

\section{Tendências de pesquisa}

Com base na reflexão teórica sobre a abordagem de redes de stakeholders, é possível inferir que as pesquisas recentes estão deslocando o foco das relações com os stakeholders centradas na organização para relações de uma rede descentralizada e com diversos atores. Além disso, alguns pesquisadores como Schneider e Sachs (2017) e Bridoux e Stoelhorst (2014) estão explorando também, como unidade de análise para os estudos, as redes de stakeholders e as identidades sociais dos membros desses grupos, indo além da análise tradicional com foco na empresa, para entender os comportamentos, as atitudes e as percepções dos indivíduos afiliados às redes de stakeholders.

Essa nova abordagem de pesquisa está também alinhada aos recentes estudos da área da estratégia (BRIDOUX e STOELHORST, 2014; SCHNEIDER e SACHS, 2017) que exploram as microfoundations para a criação de valor organizacional, e como os comportamentos cooperativos entre os stakeholders influenciam no desempenho das organizações a sua volta.

Assim, argumenta-se que conceituar os stakeholders como grupos sociais e estudar suas relações por meio das redes sociais se mostra ainda um relevante campo de estudos, considerando os mesmos argumentos apresentados por Rowley (1997) e Rowley e Moldoveanu (2003). A rede de stakeholders revela-se ainda como uma importante unidade de análise adicional, propiciando o desenvolvimento e aplicação de instrumentos de pesquisa de vários níveis para investigar o desenvolvimento de capacidades, recursos e conhecimentos, além da criação de valor, partindo da ótica das redes de stakeholders.

Diante das análises e tendências de pesquisas apresentadas, propõem-se alguns tópicos a seguir, para estudos futuros da abordagem de redes de stakeholders.

- Analisar como as redes de influência de stakeholders contribuem para a criação de valor por meio de ações estratégicas de sinergia (TANTALO e PRIEM, 2016), ou seja, ações que beneficiam vários stakeholders simultaneamente.

- Avaliar como a influência de redes de stakeholders, organizadas em torno de um interesse em comum (SCHNEIDER e SACHS, 2017), afetam as empresas e seu relacionamento com os múltiplos stakeholders.

- Descrever empiricamente como as identidades sociais dos indivíduos inseridos em redes de stakeholders modelam sua afiliação e comprometimento com relação a uma empresa focal.

- Analisar a capacidade de influência de atores que assumem diferentes papéis em relação a uma organização (ou seja, um mesmo indivíduo assumindo o papel de acionista e de consumidor, ou de acionista e de funcionário simultaneamente) na perspectiva de redes sociais.

- Desenvolver ou aprimorar modelos de criação de valor conjunta e cocriação de valor (BRIDOUX e STOELHORST, 2016) na perspectiva da cooperação em rede de múltiplos stakeholders.

- Avaliar os ganhos relacionais no engajamento de stakeholders, na perspectiva de que os indivíduos inseridos em redes desenvolvem comportamentos coletivos de participação social.

- Levantar impactos negativos na reputação organizacional advindos do comportamento dos stakeholders em redes, como boicotes e divulgação de informações negativas.

- Identificar outras abordagens que se beneficiem da inter-relação entre as redes de stakeholders, em uma perspectiva interdisciplinar. 


\section{CONSIDERAÇõES FINAIS E IMPLICAÇÕES}

A análise da abordagem de redes de stakeholders revela-se promissora para os estudos que buscam investigar as interconexões entre múltiplos stakeholders e sua influência nas organizações. Observou-se que esta abordagem tem relação com a teoria de redes sociais, com a teoria dos stakeholders e com a teoria organizacional, e para cada corrente teórica há autores proeminentes que influenciam as publicações.

A abordagem de redes de stakeholders apresenta vantagens para o campo teórico, empírico e gerencial. Contribui para a definição dos limites de uma rede organizacional, o que pode levar a um gerenciamento mais efetivo dos stakeholders. Ela também se destaca pelo potencial de criação de valor e de troca de recursos entre os atores, propiciando cooperação e sinergia. Finalmente, a abordagem tem um papel importante no processo estratégico, dado que a influência dos atores em rede tem impacto na adoção de estratégias.

As considerações levantadas por este estudo basearam-se nos trabalhos teóricos e empíricos publicados sobre redes de stakeholders nos últimos 20 anos. As proposições de Rowley (1997) são rediscutidas e, de certa forma, tangenciadas por pesquisadores, como Roloff (2008), Zeng, Chen, Dong et al. (2015), Yang e Bentley (2017), entre outros, que reconhecem a importância de uma análise aprofundada das relações entre os stakeholders. Como apresentado e discutido no framework sintético sobre a evolução da abordagem teórica de redes de stakeholders, ambos os artigos empíricos analisados, a exemplo de Garriga (2009), Fassin, Cole e Freeman (2017), e também os artigos teóricos que se debruçam com maior afinco na utilização do Modelo de Rowley, como Schneider e Sachs (2017), Sciareli e Tani (2013) e Ali (2017), reconhece-se a importância do uso da análise de rede social para examinar como a estrutura da rede de stakeholders e a posição de cada stakeholder dentro dessa estrutura podem influenciar a organização.

Com base na reflexão teórica sobre a abordagem de redes de stakeholders, é possível inferir que as pesquisas recentes estão deslocando o foco das relações com os stakeholders centradas na organização, para as relações de uma rede descentralizada e com diversos atores. Esta questão reflete a interconexão entre os campos teóricos de redes organizacionais com os estudos apoiados na abordagem da teoria dos stakeholders, conforme observado nos estudos de Provan, Fish e Sydow (2007) e Yang e Bentley (2017). Evidencia-se que há uma tendência de estudos sobre as redes de stakeholders que vão além da análise tradicional com foco na empresa, e que buscam compreender, pelas identidades sociais dos membros afiliados à rede, os seus comportamentos, as suas atitudes e percepções. Além disso, como evidenciado na proposta de tendências de pesquisa, que teve por base os estudos de Schneider e Sachs (2017) e Bridoux e Stoelhorst (2014), outras possibilidades de pesquisas emergem, principalmente com a evolução da própria teoria de stakeholders, o que tem contribuído para o elevado o número de publicações nos últimos anos.

Este estudo tem suas limitações reconhecidas. Primeiramente, como o objetivo era apresentar a evolução da abordagem de redes de stakeholders com base na proposta de Rowley $(1997,2017)$, outros estudos que tenham se desdobrado por intermédio da conjugação da teoria de redes sociais e teoria dos stakeholders, mas de forma independente à publicação de Rowley (1997), não foram incluídos nesta revisão. Embora os estudos, neste âmbito em particular, estivessem fora do escopo definido, reconhece-se que eles poderiam ser importantes para melhor compreender como estas teorias se aproximam, são operacionalizadas e contribuem para a literatura organizacional de forma geral. Em segundo lugar, o critério exclusivo de seleção de artigos revisados por pares do banco de dados ISI, na área de Business \& Management, pode ter omitido alguma pesquisa relevante. Consequentemente, recomendam-se pesquisas adicionais para investigar outros bancos de dados. Além disso, é importante implementar pesquisas com outros operadores booleanos que contemplem termos relativos a redes sociais e stakeholders.

Apesar destas limitações, reconhece-se que a contribuição desta pesquisa consiste na elaboração de um panorama teórico da abordagem de redes sociais para a análise de stakeholders, apresentando as redes de referências, as contribuições teóricas e empíricas e a tendência dos estudos recentes, vindo assim a auxiliar futuras pesquisas nessa temática. 


\section{REFERÊNCIAS}

AGLE, B. R.; MITCHELL, R. K.; SONNENFELD, J. A. Who matters to CEOs? An investigation of stakeholder attributes and salience, corporate performance, and CEO values. Academy of Management Journal, v. 42, n. 5, p. 507-525, 1999.

AHUJA, G.; SODA, G.; ZAHEER, A. The genesis and dynamics of organizational networks. Organization Science, v. 23, n. 2, p. 434448, 2012.

ALI, M. A. Stakeholder salience for stakeholder firms: An attempt to reframe an important heuristic device. Journal of Business Ethics, v. 144 , n. 1, p. 153-168, 2017.

BALESTRIN, A.; VERSCHOORE, J. Redes de Cooperação Empresarial: Estratégias de Gestão na Nova Economia. Porto Alegre: Bookman Editora, 2016.

BARNARD, C. I. The Functions of the Executive. Cambridge Mass.: Harvard University Press, 1938.

BARNEY, J. Firm resources and sustained competitive advantage. Journal of Management, v. 17, n. 1, p. 99-120, 1991.

BERGENHOLTZ, C.; WALDSTR ØM, C. Inter-organizational network studies - a literature review. Industry and Innovation, v. 18, n. 6, p. 539-562, 2011.

BERMAN, S. L. et al. Does stakeholder orientation matter? The relationship between stakeholder management models and firm financial performance. Academy of Management Journal, v. 42, n. 5, p. 488-506, 1999.

BRANDÃO, I. F.; DIÓGENES, A. S. M.; ABREU, M. C. Value allocation to stakeholder employees and its effect on the competitiveness of the banking sector. Revista Brasileira de Gestão de Negócios, v. 19, n. 64, p. 161-179. 2017.

BRIDOUX, F.; STOELHORST, J. W. Microfoundations for stakeholder theory: Managing stakeholders with heterogeneous motives. Strategic Management Journal, v. 35, n. 1, p. 107-125, 2014.

BRIDOUX, F.; STOELHORST, J. W. Stakeholder relationships and social welfare: A behavioral theory of contributions to joint value creation. Academy of Management Review, v. 41, n. 2, p. 229$251,2016$.

BURT, R. S. A note on social capital and network content. Social networks, v. 19, n. 4, p. 355-373, 1997.

CABRAL, S.; FERNANDES, A. S. A.; RIBEIRO, D. B. C. Os papéis dos stakeholders na implementação das parcerias público-privadas no Estado da Bahia. Cadernos EBAPE.BR, Rio de Janeiro, v. 14, n. 2, p. 325-339, 2016.

CALLON, M. Por uma nova abordagem da ciência, da inovação e do mercado: o papel das redes sócio-técnicas. In: PARENTE, A. (Org.). Tramas da rede: novas dimensões filosóficas, estéticas e políticas de comunicação. Porto Alegre: Sulina, 2004. p. 64-79.

CARROLL, A.B. A three-dimensional conceptual model of corporate performance. Academy of management review, v. 4, n. 4, p. $497-$ $505,1979$.

CASSAROTO FILHO, N.; PIRES, L. H. Redes de pequenas e médias empresas e desenvolvimento local: estratégia para conquista da competitividade global com base na experiência italiana. São Paulo: Atlas, 1998.

CASTELLS, M. Para o Estado-rede: globalização econômica e instituições políticas na era da informação. In: BRESSER-PEREIRA, L. C.; SOLA, L.; WILHEIM, J. (Org.). Sociedade e Estado em transformação. São Paulo: Editora UNESP, 1999. p. 147-171.

CASTELLS, M. Communication, power and counter-power in the network society. International Journal of Communication, v. 1, p. 238-266, 2007.

CASTELLS, M.; MAJER, R. V.; GERHARDT, K. B. A sociedade em rede. Lisboa: Fundação Calouste Gulbenkian, 2002.

CLARKSON, M. E. A stakeholder framework for analyzing and evaluating corporate social performance. Academy of management review, v. 20, n. 1, p. 92-117, 1995.

CONTRACTOR, F. J.; LORANGE, P. Why should firms cooperate? The strategy and economics basis for cooperative ventures. In: CONTRACTOR, F.; LORANGE, P. (Eds.). Cooperative Strategies in International Business. Massachusets: Lexington Books, 1988. p. 3-30.

CROSSAN, M. M.; APAYDIN, M. A multi-dimensional framework of organizational innovation: A systematic review of the literature. Journal of Management Studies, v. 47, n. 6, p. 1154-1191, 2010.

DONALDSON, T.; PRESTON, L. E. The stakeholder theory of the corporation: Concepts, evidence, and implications. Academy of Management Review, v. 20, n. 1, p. 65-91, 1995.

FASSIN, Y.; COLLE, S.; FREEMAN, R. E. Intra-stakeholder alliances in plant-closing decisions: A stakeholder theory approach. Business Ethics: A European Review, v. 26, n. 2, p. 97-111, 2017.

FREEMAN, R. E. Strategic management: A stakeholder approach. Boston: Pitman, 1984.

FREEMAN, R. E.; EVAN, W. M. Corporate governance: A stakeholder interpretation. Journal of Behavioral Economics, v. 19, n. 4, p. $337-$ $359,1990$.

FROOMAN, J. The issue network: Reshaping the stakeholder model. Canadian Journal of Administrative Sciences, v. 27, n. 2, p. 161173, 2010

GARRIGA, E. Cooperation in stakeholder networks: firms" 'Tertius lungens' role. Journal of Business Ethics, v. 90, n. 4, p. 623-637, 2009.

GIBBS, B. H.; SINGER, J. D. Empirical knowledge on world politics: A summary of quantitative research, 1970-1991. Westport: Greenwood Pub Group, 1993.

GRANOVETTER, M. Economic action and social structure: The problem of embeddedness. American Journal of Sociology, v. 91, n. 3, p. 481-510, 1985.

GRANOVETTER, M. Society and economy: Framework and principles. Cambridge: Harvard University Press, 2017.

GRAY, B.; WOOD, D. J. Collaborative alliances: Moving from practice to theory. The Journal of Applied Behavioral Science, v. 27, n. 1, p. 3-22, 1991. 
GRIFFIN, J. J. Tracing stakeholder terminology then and now Convergence and new pathways. Business Ethics: A European Review, v. 26, n. 4, p. 326-346, 2017.

GULATI, R.; NOHRIA, N.; ZAHEER, A. Strategic networks. Strategic Management Journal, v. 21, n. 3, p. 203-215, 2000.

HACKETT, S. M.; DILTS, D. M. A systematic review of business incubation research. The Journal of Technology Transfer, v. 29, n. 1, p. 55-82, 2004.

HARRISON, J.; FREEMAN, E.; ABREU, M. C. S. Stakeholder Theory as an ethical approach to effective management: applying the theory to multiple contexts. Revista Brasileira de Gestão de Negócios, v. 17, n. 55 , p. $858-869.2015$

HARRISON, J. S.; WICKS, A. C. Stakeholder theory, value, and firm performance. Business Ethics Quarterly, v. 23, n. 1, p. 97-124, 2013.

HEUGENS, P.; VAN DEN BOSCH, F. A. J.; VAN RIEL, C. Stakeholder integration: Building mutually enforcing relationships. Business \& Society, v. 41, n. 1, p. 36-60, 2002.

HUXHAM, C.; VANGEN, S. Working together: Key themes in the management of relationships between public and non-profit organizations. International Journal of Public Sector Management, v. 9, n. 7, p. 5-17, 1996.

JACK, S. L. Approaches to studying networks: Implications and outcomes. Journal of Business Venturing, v. 25, n. 1, p. 120-137, 2010.

JENSEN, M.C. Value maximization, stakeholder theory, and the corporate objective function. Business ethics quarterly, p. 235-256, 2002.

JONES, T. M.; WICKS, A.C. Convergent stakeholder theory. Academy of management review, v. 24, n. 2, p. 206-221, 1999.

LATOUR, B. Jamais fomos modernos. São Paulo: Editora 34, 1994.

MILES, S. Stakeholder theory classification: A theoretical and empirical evaluation of definitions. Journal of Business Ethics, v. 142, n. 3 p. 437-459, 2017.

NEVILLE, B. A.; MENGUC, B. Stakeholder multiplicity: Toward an understanding of the interactions between stakeholders. Journa of Business Ethics, v. 66, n. 4, p. 377-391, 2006.

PFEFFER, J.; SALANCIK, R.G. The external control of organizations: A resource dependence perspective. 1978.

PHILLIPS, R. Stakeholder legitimacy. Business ethics quarterly, v. 13, n. 1 , p. $25-41,2003$.

PORTER, M. E. Competitive strategy: techniques for analysing industries and competitors. New York: Free Press, 1980.

POWELL, W. Neither markets nor hierarchy: network forms of organization. Res Organ Behav, 12, pp. 295-336. 1990.

PROVAN, K. G.; FISH, A.; SYDOW, J. Interorganizational networks at the network level: A review of the empirical literature on whole networks. Journal of Management, v. 33, n. 3, p. 479-516, 2007.

RIBEIRO, N. C.; ANTONIALLI, L. M.; ZAMBALDE, A. L. Análise sociométrica da estrutura da rede de propriedade intelectual de uma universidade pública. Perspectivas em Gestão \& Conhecimento, v. 5, n. 1, p. 127 146, 2015

ROBERTS, N. C.; BRADLEY, R. T. Stakeholder collaboration and innovation: A study of public policy initiation at the state level. The Journal of Applied Behavioral Science, v. 27, n. 2, p. 209-227, 1991.
ROLOFF, J. A life cycle model of multi-stakeholder networks. Business Ethics: A European Review, v. 17, n. 3, p. 311-325, 2008.

ROTH, A. L. et al. Diferenças e inter-relações dos conceitos de governança e gestão de redes horizontais de empresas: contribuições para o campo de estudos. Revista de Administração, São Paulo, v. 47, n. 1, p. 112-123, 2012.

ROWLEY, T. J. Moving beyond dyadic ties: A network theory of stakeholder influences. Academy of Management Review, v. 22, n. 4, p. 887-910, 1997.

ROWLEY, T. J. The Power of and in Stakeholder Networks. In: WASIELESKI, D. M.; WEBER, J. (Eds). Stakeholder Management. Series Business and Society 360. Emerald Publishing Limited, 2017. p. 101-122.

ROWLEY, T. J.; MOLDOVEANU, M. When will stakeholder groups act? An interest-and identity-based model of stakeholder group mobilization. Academy of Management Review, v. 28, n. 2, p. 204219, 2003

RÜHLI, E. et al. Innovation in multistakeholder settings: The case of a wicked issue in health care. Journal of Business Ethics, v. 143, n. 2, p. 289-305, 2017

SAUR-AMARAL, I. Revisão sistemática da literatura. Lisboa: Bubok, 2010.

SCARINGELLA, L.; RADZIWON, A. Innovation, entrepreneurial, knowledge, and business ecosystems: Old wine in new bottles? Technological Forecasting and Social Change, 2017. Disponível em: <https://doi. org/10.1016/j.techfore.2017.09.02>. Acesso em: 04 set. 2019.

SCHNEIDER, T.; SACHS, S. The impact of stakeholder identities on value creation in issue-based stakeholder networks. Journal of Business Ethics, v. 144, n. 1, p. 41-57, 2017

SCIARELLI, M.; TANI, M. Network approach and stakeholder management. Business Systems Review, v. 2, n. 2, p. 175-190, 2013.

SCOTT, R.W.; DAVIS, G. F. Organizations and organizing: Rational, natural and open systems perspectives. New York: Routledge, 2017.

STOCKER, F.; MASCENA, K. M. C. Orientação e gestão para stakeholders no processo de decisão organizacional. Revista de Gestão e Secretariado, GESEC, São Paulo, v. 10, n. 1, p. 167-191, jan./abr. 2019.

TANTALO, C.; PRIEM, R. L. Value creation through stakeholder synergy. Strategic Management Journal, v. 37, n. 2, p. 314-329, 2016.

WADDOCK, S.A.; GRAVES, S.B. The corporate social performancefinancial performance link. Strategic management journal, v. 18, n. 4, p. 303-319, 1997.

VAN ECK, N. J.; WALTMAN, L. Visualizing bibliometric networks. In: DING. Y.; ROUSSEAU, R.; WOLFRAM, D. (Eds.). Measuring scholarly impact. Springer International Publishing, 2014. p. 285-320.

YANG, A.; BENTLEY, J. A balance theory approach to stakeholder network and apology strategy. Public Relations Review, v. 43, n. 2, p. 267-277, 2017

ZACCARELLI, S. et al. Clusters e redes de negócios: uma nova visão para a gestão dos negócios. São Paulo: Atlas, 2008.

ZEDAN, S.; MILLER, W. Using social network analysis to identify stakeholders' influence on energy efficiency of housing. International Journal of Engineering Business Management, v. 9, p. 1-11, 2017.

ZENG, F. et al. Understanding distributor opportunism in a horizontal network. Industrial Marketing Management, v. 46, p. 171-182, 2015 


\section{Fabricio Stocker}

ORCID: https://orcid.org/0000-0001-6340-9127

Doutorando em Administração na Faculdade de Economia, Administração e Contabilidade da Universidade de São Paulo (FEA-USP), São Paulo - SP, Brasil. E-mail: fabriciostocker@usp.br

Keysa Manuela Cunha de Mascena

ORCID: https://orcid.org/0000-0002-0844-500X

Doutora em Administração pela Faculdade de Economia, Administração e Contabilidade da Universidade de São Paulo (FEA-USP); Professora do Programa de Pós-Graduação em Administração de Empresas da Universidade de Fortaleza (PPGA-UNIFOR), Fortaleza - CE, Brasil. E-mail: keysamascena@unifor.br

Ana Cláudia Azevedo

ORCID: https://orcid.org/0000-0001-8141-9265

Doutora em Administração pela Faculdade de Economia, Administração e Contabilidade da Universidade de São Paulo (FEA-USP), São Paulo - SP, Brasil. E-mail: anacazevedo@usp.br

João Maurício Gama Boaventura

ORCID: https://orcid.org/0000-0003-3070-2153

Doutor em Administração pela Universidade de São Paulo; Professor Titular na Faculdade de Economia, Administração e Contabilidade da Universidade de São Paulo (FEA-USP) São Paulo - SP, Brasil. E-mail: jboaventura@usp.br 